\title{
Schweizerische Vereinigung gegen die Osteoporose
}

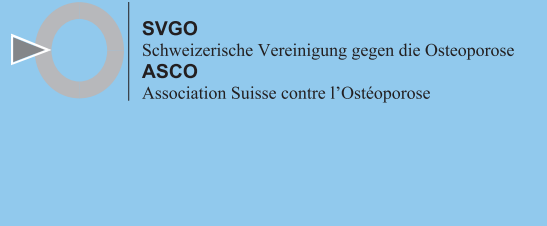

\section{Executive committee}

\section{Präsident}

Prof. Serge Ferrari

Division des Maladies Osseuses

Département des spécialités de Médecine

Rue Gabrielle-Perret-Gentil 4

1205 Genève

Tel.: + 41 (0) 223729960

Fax: + 41 (0) 223829973

E-Mail: Serge.Ferrari@unige.ch

Vize-Präsident (president-elect)

Prof. Heike A. Bischoff-Ferrari, DrPH

Klinikdirektorin, Klinik für Geriatrie, Universitäts-Spital Zürich

Klinik für Geriatrie,

Universitäts-Spital Zürich,

RAE B, Rämistrasse 100

8091 Zürich

Tel.: + 41 (0) 442552699

E-Mail: heike.bischoff@usz.ch,

heikeabischoff@aol.com

\section{Past-Präsident (ex officio)}

Prof. Christian Meier

Aeschenvorstadt 57

4051 Basel

Tel.: + 41 (0) 612649797

Fax: + 41 (0) 612649796

E-Mail: christian.meier@unibas.ch

\section{Sekretär}

Frau Dr. med. Diana Frey

Klinik für Rheumatologie,

Universitätsspital Zürich

Gloriastrasse 25

8091 Zürich

Tel.: + 41 (0) 442554212

Fax: + 41 (0) 442554565

E-Mail: diana.frey@usz.ch

\section{Kassier}

Prof. Kurt Lippuner

Universitätspoliklinik für Osteoporose

Inselspital

3010 Bern

Tel.: + 41 (0) 316323128

Natel.: + 41 (0) 793664543

Fax: + 41 (0) 316320328

E-Mail: kurt.lippuner@insel.ch

Präsident SBMS (ex officio)

Prof. Patrick Ammann

Service des Maladies Osseuses

Hôpital Cantonal

1211 Genève 14

Tel.: + 41 (0) 223829960

Fax: + 41 (0) 223829973

E-Mail: patrick.ammann@hcuge.ch

\section{Scientific committee}

Prof. Olivier Lamy

Service de Médecine Interne

Rue du Bugnon 46

1011 Lausanne

Tel.: + 41 (0) 213140963

Fax: +41 (0) 213140871

E-Mail: olivier.lamy@chuv.ch

Prof. Norbert Suhm

Klinik für Traumatologie und Orthopädie

Universitätsspital Basel

Spitalstrasse 21

4031 Basel

Tel.: + 41 (0) 613287352

E-Mail:norbert.suhm@usb.ch

Frau Dr. med. Bérengère Rozier Aubry

Service de rhumatologie et Centre

des maladies osseuses

Département de l' Appareil Locomoteur

Hopital orthopédique

Av. Pierre Decker 4

1011 Lausanne

Tel.: + 41 (0) 214141524

Fax: + 41 (0) 213144547

E-Mail: berengere.aubry@chuv.ch
Marianne Rohrbach

PD Dr. med. et phil. nat M. Rohrbach

Leitende Ärztin, Abteilung Stoffwechsel

Universitäts-Kinderspital Zürich -

Eleonorenstiftung

Steinwiesstrasse 75

8032 Zürich

Tel.: + 41 (0) 442668408

Fax: + 41 (0) 442667167

E-Mail: marianne.rohrbach@kispi.uzh.ch

Prof. Thierry Chevalley

Médecin adjoint agrégé

Responsable unité de gériatrie

de liaison $C-R$

Service de gériatrie - DMIRG - HUG

Rue Gabrielle Perret Gentil 4

1205 Genève

Tél.: + 41 (0) 223723880

E-Mail: thierry.chevalley@hcuge.ch

Dr. med. Fabio Cattaneo

Via Soldino 7

6900 Lugano

Tel.: + 41 (0) 919603030

Fax: + 41 (0) 919603039

E-Mail: fabio.cattaneo@monucco.ch

PD Dr. med. Albrecht Popp

Department of Osteoporosis,

Inselspital, Bern University Hospital,

University of Bern

3010 Bern

E-Mail: Albrecht.Popp@insel.ch

Dr. med. Siegrist Jehle

Osteoporose Zentrum Klinik St. Anna

St. Anna im Bahnhof Zentralstrasse 1 6003 Luzern

Tel.: + 41 (0) 415566191

Fax: + 41 (0) 415566192

E-Mail: osteoporosezentrum.stanna@ hirslanden.ch 
Dr. med. Maki Kashiwagi

Fachärztin für Gynäkologie und

Geburtshilfe

Direktorin/Inhaberin gynosense AG

Bankstrasse 8

8610 Uster

Tel.: + 41 (0) 434442000

Fax: + 41 (0) 434442001

E-Mail: maki.kashiwagi@gynosense.ch 